\title{
Metal About the Hip and Artifact Reduction Techniques: From Basic Concepts to Advanced Imaging
}

\author{
Iman Khodarahmi, MD, PhD ${ }^{1}$ Amanda Isaac, MBChB, MRCS, FRCR ${ }^{2,3}$ Elliot K. Fishman, $\mathrm{MD}^{4}$ \\ Danoob Dalili, MBBS, FRCR $2,3,4$ Jan Fritz, $\mathrm{MD}^{4}$
}

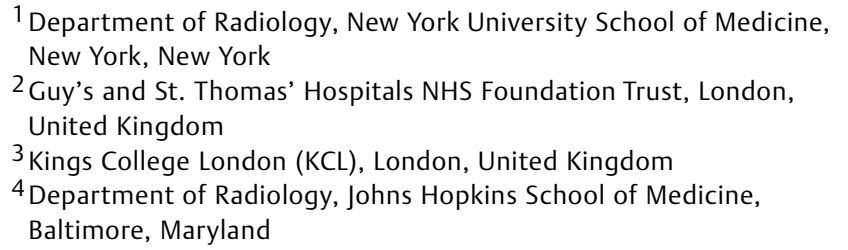

Address for correspondence Jan Fritz, MD, Department of Radiology, Johns Hopkins School of Medicine, 733 N. Broadway, Baltimore, MD 21205 (e-mail: jfritz9@jhmi.edu).

Semin Musculoskelet Radiol 2019;23:e68-e81.

\begin{abstract}
Promising outcomes of hip replacement interventions in this era of aging populations have led to higher demands for hip arthroplasty procedures. These require effective methods and techniques for the detection of postoperative outcomes and complications. Based on the presence or absence of radiographic findings, magnetic resonance imaging (MRI) and computed tomography (CT) may be required to detect and further characterize different causes of failing implants. Yet metal-related artifacts degrade image quality and pose significant challenges for adequate image quality. To mitigate

Keywords

- MRI

- CT

- implant

- hip arthroplasty

- dual energy

- metal artifact reduction such artifacts in MRI, a set of techniques, collectively known as metal artifact reduction sequence (MARS) MRI, were developed that optimize the framework of the conventional pulse sequences and exploit novel multispectral and multispatial imaging methods such as Slice Encoding for Metal Artifact Correction (SEMAC) and MultiAcquisition Variable-Resonance Image Combination (MAVRIC). Metal-induced artifacts on CT can be effectively reduced with virtual monochromatic reconstruction of dualenergy $\mathrm{CT}$ data sets, metal artifact reduction reconstruction algorithms, and postprocessing image visualization techniques.
\end{abstract}

Improvements in implant design and surgical techniques, along with higher demands for maintained mobility despite older age, have drastically increased the number of patients undergoing hip replacement surgery. As one of the surgical procedures with the best outcome, total hip arthroplasty (THA) is estimated to reach an annual rate of 570,000 in the United States $^{1}$ and 324,000 in the United Kingdom ${ }^{2}$ by 2030.

Complications associated with THA may originate from the implant itself, surrounding osseous structures, periprosthetic soft tissues, or synovial reaction. ${ }^{3}$ Although complication rates are low, ${ }^{4}$ the high prevalence of the hip replacement procedures will result in an overall growing number of patients with complications that will be encountered in daily radiology practice.

Issue Theme Hip and Advanced Musculoskeletal Imaging; Guest Editors, Vasco V. Mascarenhas, MD, MBA and Alberto Vieira, MD
Conventional radiography is the primary imaging tool for the routine surveillance of patients following hip replacement surgery and to investigate symptomatic individuals. Crosssectional imaging is reserved for further characterization of radiographic abnormalities or investigation of radiographically occult complications. Owing to recent developments in imaging techniques, an accurate diagnosis of THA complications in their early stages has now become feasible.

In this article, we review the basis of metal artifacts caused by THA implants on magnetic resonance imaging (MRI) and computed tomography (CT) and explain basic and advanced metal artifact reduction (MAR) techniques, as well as practical tips and tricks to optimize CT and MR imaging of hip arthroplasty implants. 


\section{Metal-related MRI Artifacts}

Image quality in the presence of metal is impaired by the inhomogeneity of the static $\left(B_{0}\right)$ and radiofrequency $\left(B_{1}\right)$ magnetic fields. The $B_{1}$ field inhomogeneity in the vicinity of metal is particularly accentuated by shielding of the radiofrequency (RF) pulse and local electric fields induced by switching gradient fields. ${ }^{5-7}$ In comparison with $\mathrm{B}_{1}$, inhomogeneity of the $B_{0}$ magnetic field has been studied more extensively and forms the basis for all MAR techniques currently available for clinical use. ${ }^{8}$ Perturbations in $\mathrm{B}_{0}$ in the vicinity of metal occur in various degrees depending on the hardware material type, orientation, and configuration, and they result in three broad categories of artifacts: spatial misregistration, signal loss, and failed fat suppression.

\section{Spatial Misregistrations}

To form an image in MRI, spatial localization of each voxel of tissue is achieved by applying position-dependent gradient fields during slice selection and readout. ${ }^{9}$ As such, the location of each spin ensemble is linearly related to the local magnetic field in that particular location, and hence to the spin precession frequency. Metal-related $\mathrm{B}_{0}$ inhomogeneities violate this linearity by altering the precession frequency of the affected spins. As a result, spins outside the slice of interest are excited during slice selection and wrongfully contribute to the formed image. Similarly, during readout, pixels are misregistered to the wrong locations along the frequency-encoding (readout) direction. Such misregistrations appear as geometric distortion, signal loss, or pileup ${ }^{9,10}$ (-Fig. 1). Because all lines of the k-space undergo a similar phase shift as a result of metal-induced $B_{0}$ perturbations,

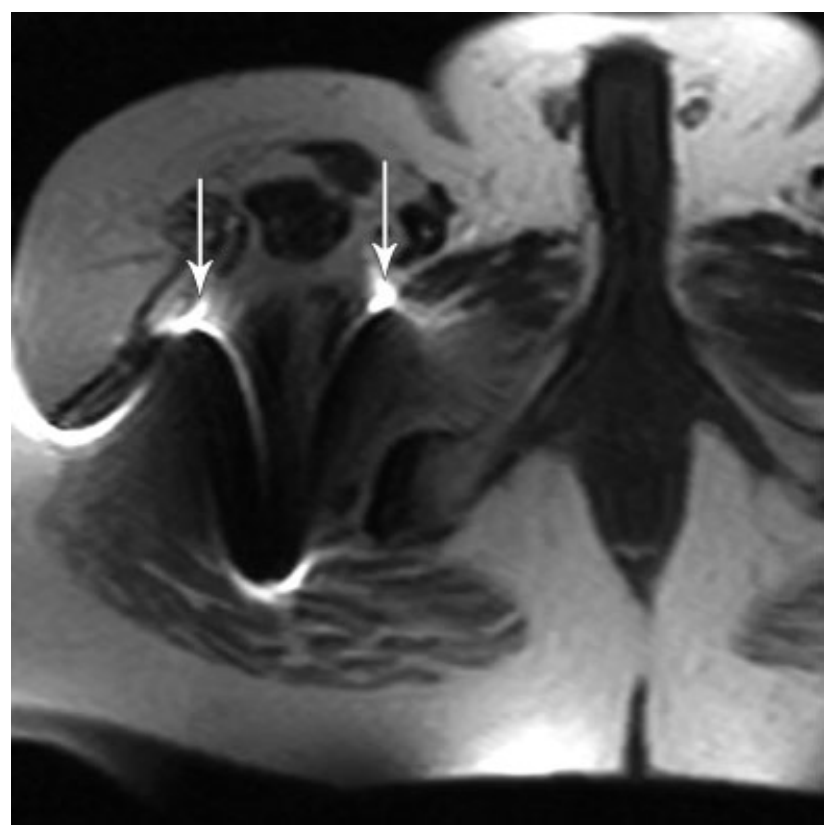

Fig. 1 Axial intermediate-weighted turbo spin-echo MR image of a 66-year-old man with right total hip replacement demonstrates geometric distortion, signal loss, and signal pileup (arrows) that occur in a frequency-encoding direction. The phase-encoding direction (right to left) is immune to such effects. unlike slice- and frequency-encoding processes, phase encoding is immune to spatial misregistrations.

\section{Signal Loss}

Significant variation of the local magnetic field within a single voxel that can occur in the vicinity of metallic implants leads to rapid dephasing and incoherence of the intravoxel spins and appears as a dark area of signal void surrounding the implant (-Fig. 1). Signal loss may also be due to failed excitation of those periprosthetic spins that resonate at a frequency outside the bandwidth of the RF pulse. ${ }^{8}$

\section{Failed Fat Suppression}

Chemical-shift-selective fat suppression benefits from the different resonance frequency of fat and water protons. By applying a saturation pulse tuned to the fat resonance frequency, it selectively suppresses the fat signal. Metal-related $\mathrm{B}_{0}$ inhomogeneity shifts the fat peak outside the frequency-specific saturation pulse, resulting in failure of fat suppression ( - Fig. 2). It may also suppress the water signal by shifting the water precession frequency into that of the fat tuned suppression pulse.

\section{Basic Strategies of MRI Metal Artifact Reduction}

\section{Imaging at Lower Field Strength Magnets}

Susceptibility-induced field inhomogeneity is linearly proportional to the field strength, and therefore one expects less metal-related artifacts at $1.5 \mathrm{~T}$ and more metal-related artifacts at 3T. However, clinical MAR techniques can be implemented on both 1.5 and 3T scanners. The higher signalto-noise ratio (SNR) of 3T imaging offers flexibility for higher

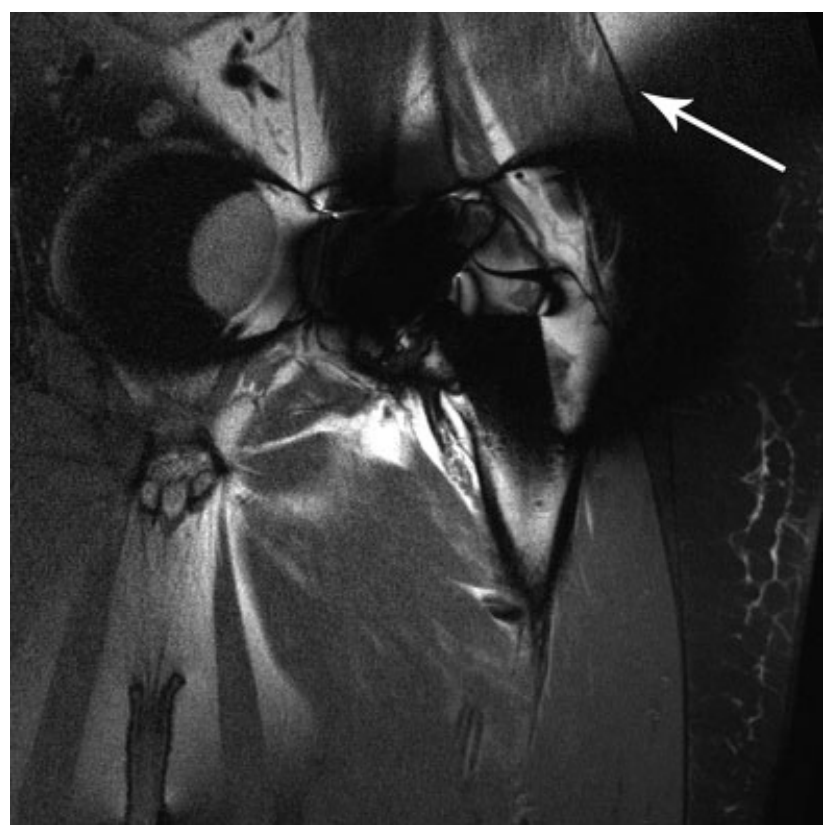

Fig. 2 Coronal T2-weighted MR image with spectral fat suppression technique of a 73-year-old man with left total hip replacement demonstrates the failure of fat suppression (arrow) around the hip implants due to shifting of the periprosthetic fat spin frequencies that consequently no longer fall within the frequency-specific saturation pulse. 
a
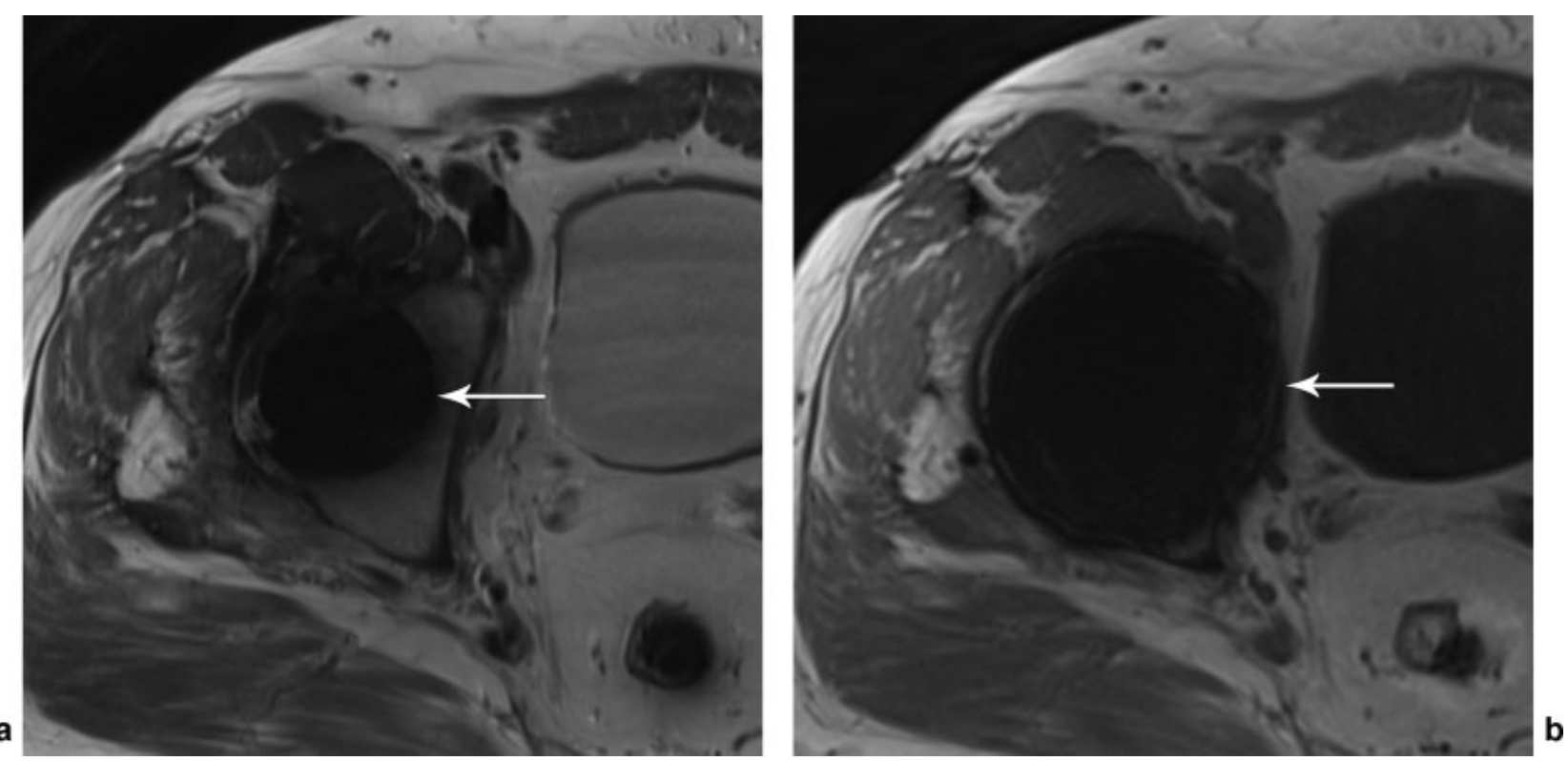

Fig. 3 Axial intermediate-weighted MR images of a 56-year-old man with a right total hip implant using high receiver bandwidth (700 Hz/pixel). (a) Turbo spin-echo and (b) gradient-echo techniques demonstrate superior performance of the spin-echo-based techniques over the gradientecho techniques in reduction of the metal-related artifacts (arrows).

image quality and together with advanced and carefully optimized MAR methods make reasonable artifact reduction possible at $3 \mathrm{~T}$ as well.

\section{Spin-echo-based Pulse Sequences}

Spin-echo-based pulse sequences effectively mitigate signal loss due to intravoxel dephasing by applying a 180-degree pulse to refocus the dephasing spins ( - Fig. 3 ). ${ }^{10}$

\section{Selection of Phase and Frequency-Encoding Directions}

Because phase encoding is unaffected by spatial misregistrations, the user may swap the phase- and frequency-encoding directions with the aim of displacing the artifacts in a direction that causes less tissue obscuration. This, however, may occur in time penalties due to the new need for phase oversampling to overcome potential wrap artifacts.

\section{High Receiver Bandwidth}

Spin-echo class pulse sequences can be optimized for MAR by increasing the bandwidth of the receiver that reduces the number of voxels across the signal displacement extends (-Fig. 4). Increasing the receiver bandwidth additionally results in improved edge sharpness but also lower SNRs. ${ }^{11}$ - Table 1 shows the Johns Hopkins MRI protocol for patients with hip arthroplasty, composed of high bandwidth turbo spinecho pulse sequences.

\section{High Radiofrequency Pulse Bandwidth}

Similar to the receiver bandwidth, increasing the bandwidth of the excitation pulse decreases the number of slices across which the signal misregistrations propagates. This comes with a penalty of increased specific absorption rate (SAR) deposition.

\section{Imaging with Thinner Slices}

Assuming fixed RF pulse bandwidth, thinner slices are achieved by applying stronger slice-selection encoding gradients. Sharing the same concept with high bandwidth RF pulses, this results in less metal-related artifacts. Although there is no increase in SAR, thinner slices are associated with decreasing SNR.

\section{Increasing the Image Matrix Size}

Increased matrix size results in smaller voxels associated with reduced intravoxel signal loss and increased conspicuity of metal-related artifacts, both resulting in improved

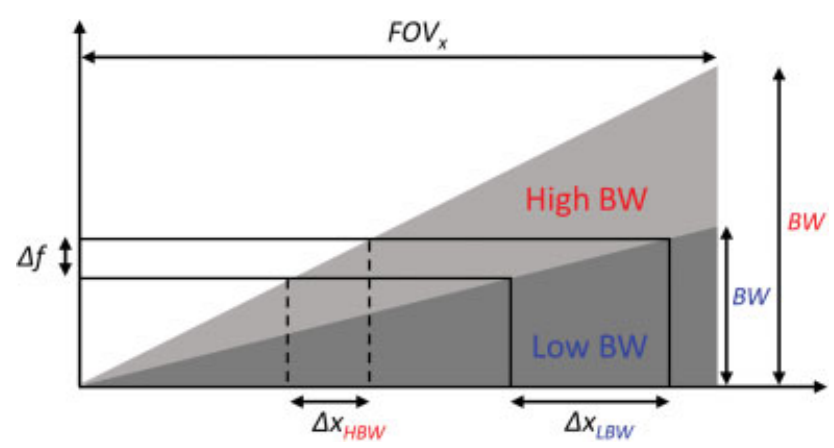

Fig. 4 Schematic demonstration of the effect of receiver bandwidth on signal displacement in the frequency-encoding dimension. Metalinduced magnetic field inhomogeneities cause a shift of the precession frequency $(\Delta f)$ of spins at a particular location. At low receiver bandwidths, this frequency shift results in a signal displacement $\left(\Delta x_{\mathrm{LBW}}\right)$. The same frequency shift $(\Delta f)$ results in smaller signal displacement at high receiver bandwidths $\left(\Delta x_{\mathrm{HBW}}\right)$. The same principle applies to excitation pulse bandwidth and displacements along the slice-selection dimension. BW, bandwidth; FOV $x$ = field of view in the frequency $(x)$ direction; HBW, high bandwidth; LBW, low bandwidth. 
Table 1 Conventional metal artifact reduction protocol for MR imaging of hip arthroplasty implants

\begin{tabular}{|l|l|l|l|l|l|l|l|}
\hline Parameters & $\begin{array}{l}\text { Coronal } \\
\text { IW TSE }\end{array}$ & $\begin{array}{l}\text { Coronal } \\
\text { STIR TSE }\end{array}$ & $\begin{array}{l}\text { Sagittal } \\
\text { IW TSE }\end{array}$ & $\begin{array}{l}\text { Sagittal } \\
\text { STIR TSE }\end{array}$ & $\begin{array}{l}\text { Axial } \\
\text { IW TSE }\end{array}$ & $\begin{array}{l}\text { Axial } \\
\text { STIR TSE }\end{array}$ & $\begin{array}{l}\text { Axial } \\
\text { T1 TSE }^{a}\end{array}$ \\
\hline TE/TR, ms & $30 / 3,800$ & $6.3 / 3,000$ & $30 / 3,800$ & $6.3 / 3,000$ & $30 / 3,800$ & $7 / 3,000$ & $6.8 / 650$ \\
\hline $\begin{array}{l}\text { Receiver bandwidth, } \\
\text { Hz/pixel }\end{array}$ & 504 & 501 & 504 & 501 & 504 & 510 & 504 \\
\hline $\begin{array}{l}\text { No. of slices/ } \\
\text { Flip angle, degrees }\end{array}$ & $27 / 150$ & $21 / 140$ & $31 / 150$ & $21 / 140$ & $35 / 150$ & $31 / 140$ & $25 / 140$ \\
\hline Field of view, mm ${ }^{2}$ & $270 \times 270$ & $300 \times 300$ & $270 \times 270$ & $300 \times 300$ & $230 \times 230$ & $230 \times 230$ & $300 \times 300$ \\
\hline Matrix & $320 \times 70 \%$ & $256 \times 80 \%$ & $320 \times 70 \%$ & $256 \times 80 \%$ & $320 \times 80 \%$ & $256 \times 80 \%$ & $205 \times 70 \%$ \\
\hline Slice thickness/Gap, mm & $3.5 / 0$ & $4 / 0$ & $3.5 / 0$ & $4 / 0$ & $4 / 0$ & $4.5 / 0.4$ & $5 / 0$ \\
\hline $\begin{array}{l}\text { No. of excitations/ } \\
\text { Concatenations }\end{array}$ & $3 / 1$ & $2 / 2$ & $3 / 1$ & $2 / 2$ & $3 / 1$ & $3 / 1$ & $1 / 1$ \\
\hline Acceleration factor & 2 & 2 & 2 & 2 & 2 & $2: 45$ & $2: 56$ \\
\hline Acquisition time, min:s & $3: 31$ & $3: 44$ & $3: 31$ & $3: 56$ & $2: 45$ \\
\hline
\end{tabular}

Abbreviations: IW, intermediate weighted; MR, magnetic resonance; STIR, short tau inversion recovery; TE, echo time; TR, repetition time; TSE, turbo spin echo.

${ }^{a}$ Optional use for pre- and postcontrast gadolinium-enhanced MRI.

image quality. ${ }^{12}$ It has no or minimal effect on in-plane distortions if other scan parameters are left unchanged. Similarly, increasing the echo train length has no direct effect on MAR. ${ }^{13}$ However, platform-specific associated effects due to changes to the receiver bandwidth in the background may result in apparent MAR effects.

\section{Using Short Tau Inversion Recovery and Dixon Methods for Fat Suppression}

Short tau inversion recovery (STIR) is a reliable technique for achieving homogeneous fat suppression of tissues surrounding metal implants. Acting based on the different T1 values of water and fat, STIR is immune to metal-induced field perturbations. ${ }^{14}$ In the presence of metal, residual failed fat suppression in STIR can be eliminated by matching of the bandwidths of the inversion and excitation pulses. ${ }^{15,16}$ Despite its superior fat suppression, STIR is of limited value in postcontrast fat suppression because contrastenhanced tissues may also be nulled due to their reduced T1 relaxation times. Dixon-based techniques acquire in- and opposed-phase images and allow for secondary water-only and fat-only image reconstructions. Compared with STIR, the fat suppression ability of Dixon in the presence of metal is inferior. ${ }^{17}$ However, it can facilitate successful postcontrast MRI. In the absence of patient motion, postprocessing subtraction of the pre- and postcontrast T1-weighted images with identical image parameters results in the most accurate fat suppression when metal implants are present (-Fig. 5, - Table 1). ${ }^{18}$

\section{Advanced Techniques of MRI Metal Artifact Reduction}

\section{View Angle Tilting}

Metal-related signal displacement along the slice-selection and frequency-encoding directions is proportional to the corresponding gradient field strengths in these directions. View angle tilting (VAT) applies this principle to decrease inplane misregistrations by replaying the slice-selection gradient field during readout ${ }^{19}$ ( - Fig. 6). This added gradient field tilts the readout direction, with the slope of the tilt being the ratio of slice selection to frequency-encoding gradient fields,
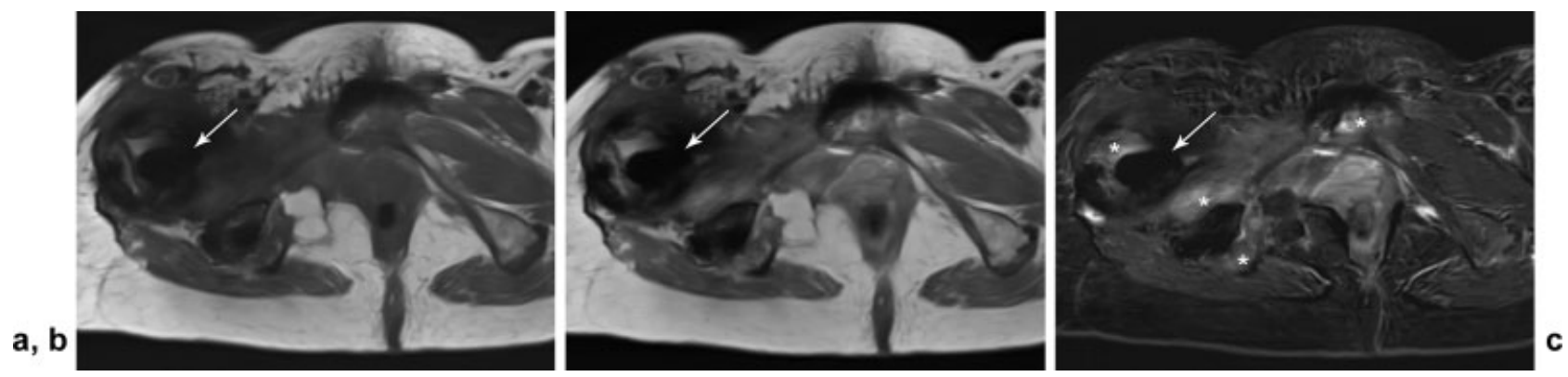

Fig. 5 Axial T1-weighted (a) precontrast and (b) postcontrast SEMAC turbo spin-echo (TSE) MR images of a 62-year-old patient with right total hip replacement (arrows) demonstrate the improved visualization of areas of contrast enhancement on the subtraction image (c, asterisk), created through the subtraction of the precontrast (a) from the postcontrast (b) image. 


\section{(a) Distortion from metal artifact}

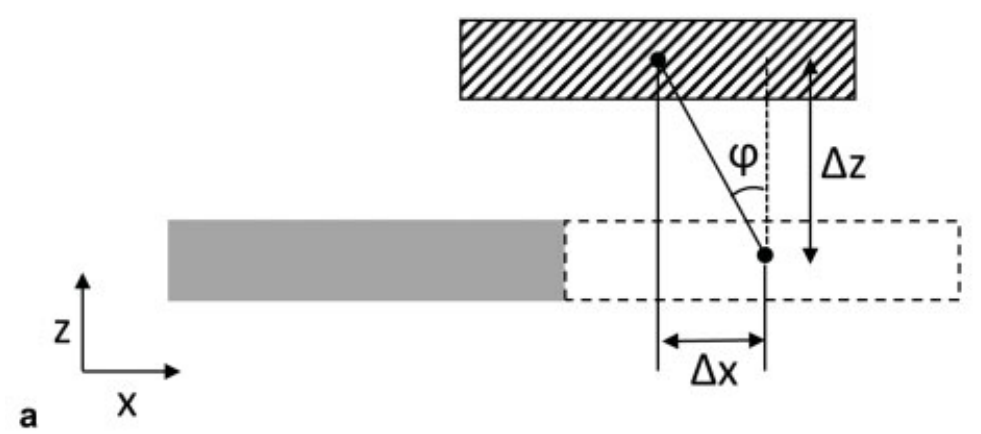

\section{(b) Conventional readout}

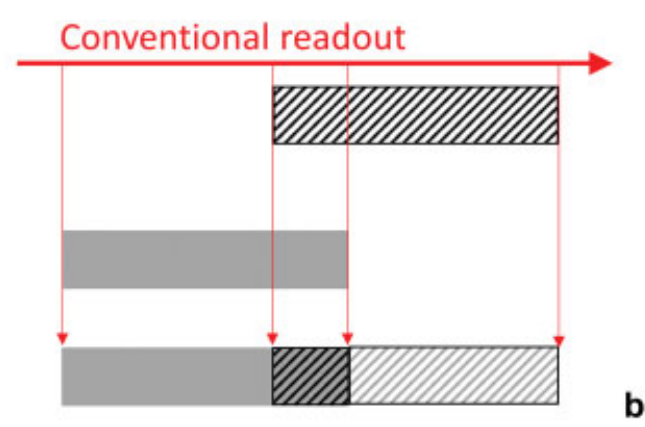

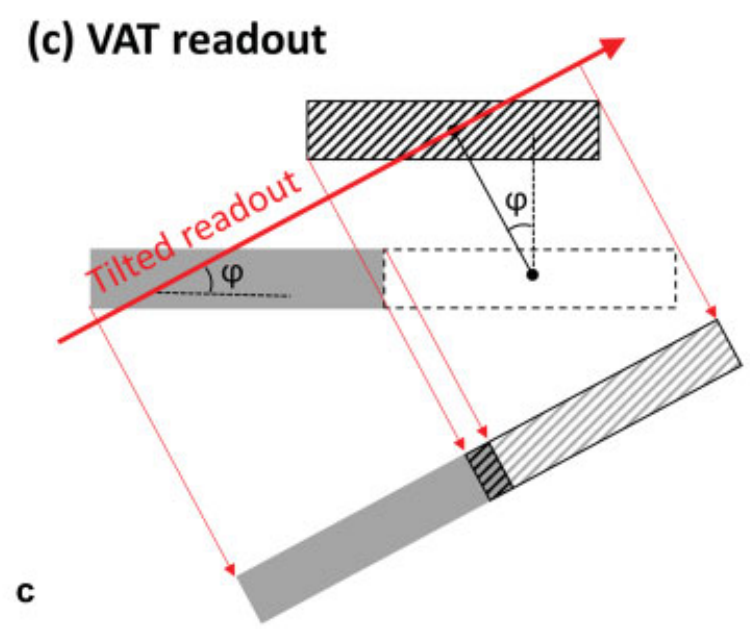

Fig. 6 Schematic illustration of the view angle tilting (VAT) technique. (a) Two adjacent blocks of tissue are represented as solid gray and white. The solid gray block is not affected by metal-related field distortions; the adjacent white block is misregistered to a new position (cross-hatched). In-plane and through-plane displacements along the readout (x) and slice-selection (z) directions are shown by $\Delta x$ and $\Delta z$, respectively. The ratio of displacement $(\tan (\phi)=\Delta x / \Delta z)$ is equal to Gs/Gf, where Gs and Gf represent slice selection and readout gradient fields, respectively. (b) In conventional imaging (b), readout in $x$ direction results in remarkable in-plane signal displacement, shown as the overlap between the two blocks, whereas with the use of VAT (c), replaying the slice-selection gradient during readout practically tilts the readout direction at the similar angle of $\phi$. The image acquired with this tilt results in less apparent in-plane displacements, although it comes at the expense of the introduction of blurring at the junction of the two blocks.

making it parallel to the spatial signal displacements. Accordingly, in-plane signal distortions are mitigated, albeit at the expense of degrees of blurring (- Fig. 7). ${ }^{20}$ From a practical standpoint, when adequate artifact reduction is achieved at increased receiver bandwidths $>500 \mathrm{~Hz} /$ pixel, application of VAT may not result in additional MAR given its image blurring effect ( $\mathbf{- F i g . 7 )})^{8}$ The combination of VAT and isotropic threedimensional (3D) fast spin-echo sequences, such as sampling perfection with application-optimized contrasts by using different flip angle evolutions (SPACE; Siemens Healthineers, Erlangen, Germany), has effectively reduced metal artifacts about a variety of implant types. ${ }^{21,22}$ However, 3D imaging of a sizable anatomical region such as the hip joint remains challenging due to time-consuming oversampling requirements in the two phase-encoding directions.

\section{Slice Encoding Metal Artifact Correction}

Slice Encoding for Metal Artifact Correction (SEMAC) is a two-dimensional (2D) imaging method that adds multiple spatial partitions to the turbo spin-echo pulse sequence to mitigate the metal-related through-plane artifacts. ${ }^{23}$ Fundamentally, SEMAC exploits an additional phase-encoding step in the slice-selection dimension to resolve the $z$ location of each distorted slice profile, similar to the way the $\mathrm{z}$-direction is encoded in 3D imaging. For each slice, images from each spatial partition are reconstructed separately and then combined into a final composite image through linear or quadratic summation (-Fig. 8). Furthermore, SEMAC may implement VAT to decrease in-plane distortions. Compared with the conventional techniques, SEMAC has proven more efficient at metal-related artifact reduction (-Fig. 7). ${ }^{24,25}$ The optimal number of additional phase-encoding steps in SEMAC, known as SEMAC steps, is a compromise between the degree of artifact reduction and longer acquisition times. ${ }^{26}$ Vendors may commercialize SEMAC with different names, such as OMAR XD (Philips, Best, Netherlands) and Advanced WARP (Siemens). - Table 2 shows the Johns Hopkins MRI protocol for patients with hip arthroplasty, composed of turbo spinecho SEMAC pulse sequences. 


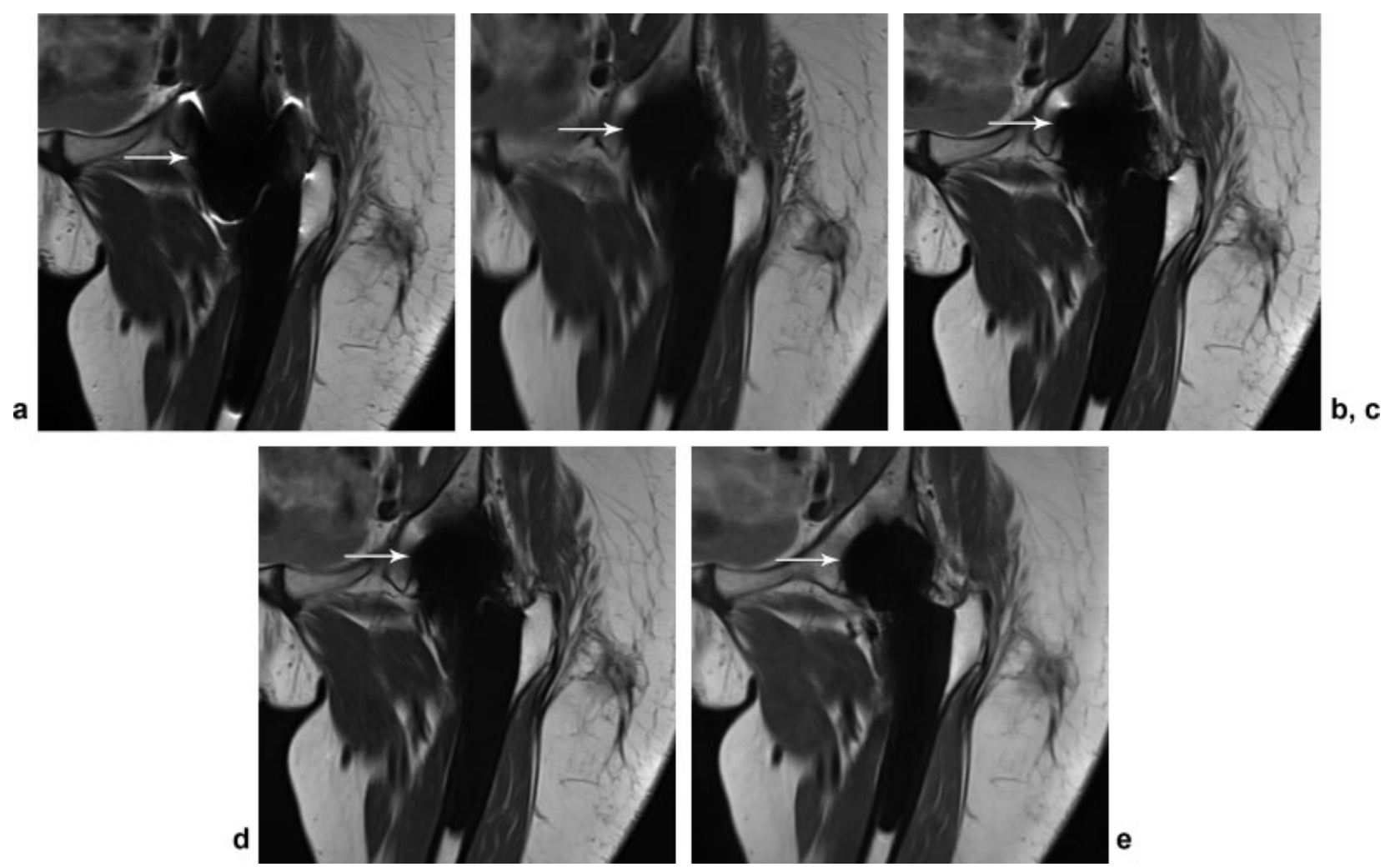

Fig. 7 Coronal intermediate-weighted turbo spin-echo MR image of a 78-year-old woman with left total hip arthroplasty implants show the application of the view angle tilting (VAT) technique for the reduction of metallic artifacts (arrows). Images a and b were acquired with a low receiver bandwidth of $150 \mathrm{~Hz} /$ pixel, (a) without and (b) with the VAT technique. In (b), the metal artifacts (arrow) are reduced by the VAT technique, at the expense of markedly increased image blurring. Images (c) and (d) were acquired with a high receiver bandwidth of $600 \mathrm{~Hz} /$ pixel, (c) without and (d) with the VAT technique. With a high receiver bandwidth, the metal artifact-reducing effect of the VAT technique is minimized (c and d, arrows); where VAT technique still introduces blurring (d), it is counteracted by the high bandwidth. (e) Image was acquired with a bandwidth of $600 \mathrm{~Hz} /$ pixel, the VAT technique, and 17 SEMAC-encoding steps, resulting in almost no metal artifacts (arrow). SEMAC and VAT technique are synergistic and achieve optimal results in combination.

\section{Multi-Acquisition with Variable-Resonance Image Combination (MAVRIC)}

Field inhomogeneity around the metal hardware causes spin precession at a wide range of frequencies, of which only a small subset, also known as "on-resonant spins," are excited by the RF pulse. ${ }^{27}$ Lack of excitation of the offresonant spins results in periprosthetic signal void. In Multi-Acquisition with Variable-Resonance Image Combination (MAVRIC), this problem was addressed by splitting each excitation into multiple frequency bins (known as spectral bins) with discrete offsets in central frequencies. For each frequency bin, a sub-image is acquired with a similar offset in the readout frequency. ${ }^{28}$ The resultant subimages are then combined through a sum-of-squares or maximum-intensity projection scheme. ${ }^{29}$ General Electric (GE, Milwaukee, WI) platforms may implement the MAVRIC-SL (MAVRIC-Selective) variant, a hybrid form of MAVRIC and SEMAC. ${ }^{30}$ Lack of slice selectivity is a major drawback of MAVRIC, requiring inflexible and time-consuming 3D imaging. Recently, a rapid and flexible 2D version of MAVRIC was proposed that excites a limited slice and spectral region using gradient reversal between excitation and refocusing pulses. ${ }^{31}$

\section{Acquisition Time Considerations}

Although multispectral (MAVRIC) and multispatial (SEMAC) imaging techniques have substantially mitigated metalrelated artifacts, this improved image quality is coupled with longer scan durations, owing to additional spectral bins in MAVRIC and spatial partitions in SEMAC. To bring the acquisition times below the clinically viable levels, various acceleration algorithms such as partial Fourier encoding and parallel imaging were implemented. ${ }^{30,32}$ Further acceleration has been achieved recently using compressed sensing (CS) that saves time by pseudo-random sampling of the k-space and retrieves the lost data through iterative image reconstruction. This technique was combined successfully with both MAVRIC $^{33}$ and SEMAC. ${ }^{34-37}$ With preserved image quality, SEMAC has gained an eightfold acceleration by exploiting all synergies between parallel imaging and CS, resulting in acquisition times that are similar to those of turbo spin-echo pulse sequences $^{34-37}$ ( - Fig. 9). The highly efficient combination of CS and SEMAC is expected to become available for clinical practice in the near future. The optimal choice of CS-SEMAC steps and iteration parameters for visualization of periprosthetic soft tissues was determined in a recent study. ${ }^{26}$ 


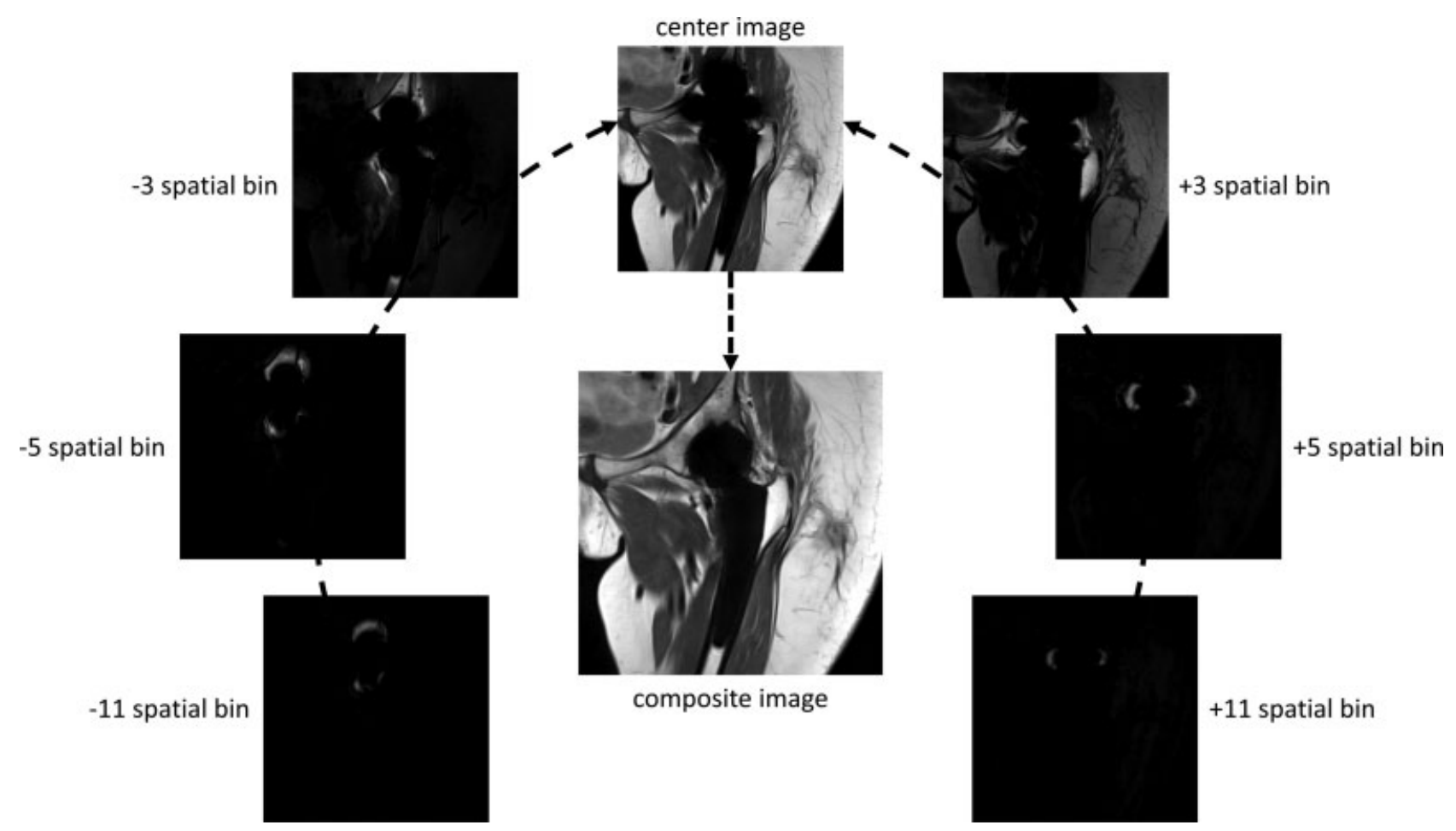

Fig. 8 Schematic diagram of the acquisition and composition process of a SEMAC-based pulse sequence with 11 encoding steps for metal artifact reduction of a left hip replacement. The final image (composite image) is a sum-of-square composition of the center image and the signals from the spatial bins that successively provide the displaced periprosthetic signal. Depending on the implant alloys, a higher number of SEMAC-encoding steps are needed to "collect" the displaced signals.

Table 2 SEMAC metal artifact reduction protocol for MR imaging of hip arthroplasty implants

\begin{tabular}{|l|l|l|l|l|l|l|l|}
\hline Parameters & $\begin{array}{l}\text { Coronal } \\
\text { IW TSE }\end{array}$ & $\begin{array}{l}\text { Coronal } \\
\text { STIR TSE }\end{array}$ & $\begin{array}{l}\text { Sagittal } \\
\text { IW TSE }\end{array}$ & $\begin{array}{l}\text { Sagittal } \\
\text { STIR TSE }\end{array}$ & $\begin{array}{l}\text { Axial } \\
\text { IW TSE }\end{array}$ & $\begin{array}{l}\text { Axial } \\
\text { STIR TSE }\end{array}$ & $\begin{array}{l}\text { Axial } \\
\text { T1 TSE }^{a}\end{array}$ \\
\hline TE/TR, ms & $32 / 2,800$ & $6.8 / 3,180$ & $32 / 3,000$ & $6.8 / 3,180$ & $32 / 3,360$ & $6.8 / 4,660$ & $6.8 / 650$ \\
\hline No. of SEMAC steps & 13 & 11 & 13 & 11 & 13 & 11 & 11 \\
\hline $\begin{array}{l}\text { Receiver bandwidth, } \\
\text { Hz/pixel }\end{array}$ & 504 & 501 & 504 & 501 & 504 & 501 & 504 \\
\hline $\begin{array}{l}\text { No. of slices/ } \\
\text { Flip angle, degrees }\end{array}$ & $27 / 140$ & $21 / 140$ & $31 / 140$ & $21 / 140$ & $35 / 140$ & $31 / 140$ & $25 / 140$ \\
\hline Field of view, mm & $270 \times 270$ & $300 \times 300$ & $270 \times 270$ & $300 \times 300$ & $270 \times 270$ & $300 \times 300$ & $300 \times 300$ \\
\hline Matrix & $320 \times 70 \%$ & $256 \times 80 \%$ & $320 \times 70 \%$ & $256 \times 80 \%$ & $320 \times 75 \%$ & $256 \times 80 \%$ & $192 \times 70 \%$ \\
\hline Slice thickness/Gap, mm & $3.5 / 0$ & $4 / 0$ & $3.5 / 0$ & $4 / 0$ & $4 / 0$ & $4 / 0$ & $5 / 0$ \\
\hline $\begin{array}{l}\text { No. of excitations/ } \\
\text { Concatenations }\end{array}$ & $1 / 1$ & $1 / 1$ & $1 / 1$ & $1 / 1$ & $1 / 1$ & $1 / 1$ & $1 / 1$ \\
\hline $\begin{array}{l}\text { Turbo factor/ } \\
\text { Acceleration factor }\end{array}$ & $11 / 3$ & $9 / 3$ & $11 / 3$ & $9 / 3$ & $11 / 3$ & $9 / 3$ & $4 / 3$ \\
\hline Acquisition time, min:s & $7: 12$ & $7: 14$ & $7: 20$ & $7: 36$ & $6: 51$ & $7: 29$ \\
\hline
\end{tabular}

Abbreviations: IW, intermediate weighted; STIR, short tau inversion recovery; TE, echo time; TR, repetition time; TSE, turbo spin echo. ${ }^{a}$ Optional use for pre- and postcontrast gadolinium-enhanced MRI.

\section{Metal-related CT Artifacts}

Metal artifacts (- Fig. 10) are seen in different grades of severity due to the eclectic range of metals, shapes, and sizes used for hip arthroplasty implants. ${ }^{38}$ These artifacts limit the accuracy for assessment of the bones, bone-metal interface, and soft tissue structures, thus may render the images unreliable in some cases. This is confounded by the fundamental nature of CT imaging, where multiplanar reconstructions are all extrapolated from single-image 

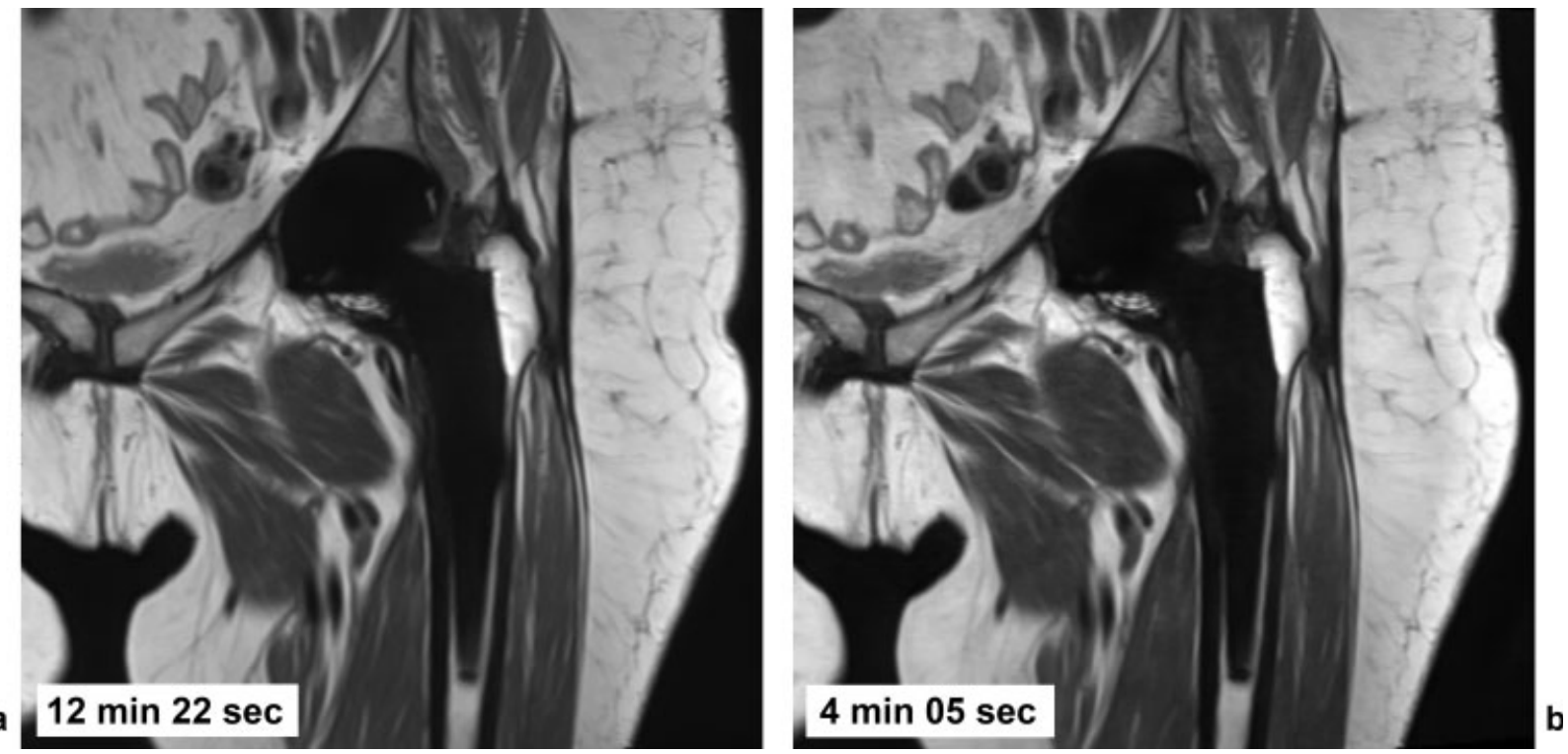

Fig. 9 Coronal intermediate-weighted MR images of a 62-year-old woman with left total hip arthroplasty implants. Comparison of conventional SEMAC with (a) threefold parallel imaging acceleration and (b) compressed-sensing SEMAC with factor 8 acceleration with otherwise the same pulse sequence parameters including 19 SEMAC-encoding steps shows similar image quality, whereas the acquisition times are threefold different.

acquisitions - unlike independent multiplanar acquisitions of MR imaging.

Contributors to metal artifacts include several factors 39 that may result in reconstruction errors near metal implants due to corrupted data, ultimately resulting in an inaccurate representation of tissues and possibly of abnormalities. These effects are broadly categorized into four main domains that overlap in their underlying etiology and effects including beam hardening, scatter, quantum noise and photon starvation, and edge effects.

\section{Beam Hardening}

Beam hardening artifacts appear as dark streaks between heavily attenuating structures, secondary to abrupt X-ray

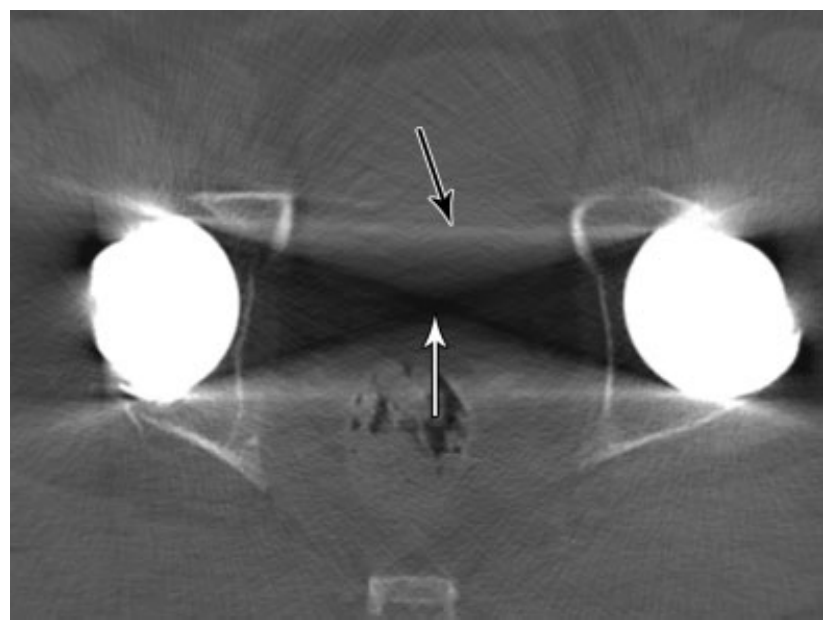

Fig. 10 Axial computed tomography image of the pelvis in a patient with bilateral total hip arthroplasty implants demonstrates beam hardening and scatter artifacts projecting as black (white arrow) and white (black arrow) streaks, respectively. beam inhomogeneities and polychromatic scatter caused by metal implants. This effect can be explained by the high density and high atomic numbers of metals used for hip replacements when compared with background bone and soft tissues. As X-ray beams pass through metal implants, the photon flux decreases with fewer low-energy photos and much more numerous high-energy photons, resulting in "hardening" of the X-ray beam. ${ }^{40,41}$

\section{Scatter}

Increased scatter of these high-energy photon beams confound standard reconstruction algorithms that ultimately results in incorrect registration. The scattered photons add to the measured intensity and lead to an underestimation of the absorption and thus to dark streaks in the image, where white streaks are caused by an overestimation of the absorption. ${ }^{42}$

\section{Quantum Noise and Photon Starvation}

Quantum noise refers to the statistical uncertainty of low photon flux due to the quantum nature of the photon counts. It manifests as random bright and dark streaks particularly appearing along the direction of highest attenuation. Photon starvation can be seen in high-density metals and in metals with a high atomic number. It leads to low photon counts and thus to increased noise and missing projection data. The background signal of the detector also adds to the noise level when no photons are detected at all. ${ }^{40,43}$

\section{Edge Effects}

Edge effects are observed at sharp edges between high and low attenuating tissues. This effect also contributes to nonlinear partial volume and misregistrations of data. ${ }^{43}$ 


\section{Basic Strategies of CT Metal Artifact Reduction}

Multiple basic steps may be applied to data acquisition, image reconstruction, and image visualization to improve the quality of CT images around hip arthroplasty implants. ${ }^{44}$

Increasing the tube voltage and current are recognized standard techniques to reduce the magnitude of metal artifacts by allowing for more penetration power and photons, respectively, resulting in less scatter and absorption and minimizing heterogeneity in the exiting photon beam. This targets scatter, noise, and photon starvation artifacts at the expense of an increased radiation dose. ${ }^{44}$

Reducing the detector size reduces the total scatter registered, thus minimizing overall scatter artifacts. Similarly, using narrow collimation can reduce the partial volume effects and simultaneously minimize scatter-related artifacts. ${ }^{45,46}$

Patient positioning, for example with bilateral hip arthroplasty implants not completely aligned parallel to each other, and, where applicable, gantry tilt, which acts along similar principles as the VAT MRI acquisition technique, may also reduce artifacts in specific scenarios. During acquisition, acquiring the largest number of projections per rotation may help reduce the extent of aliasing and undersampling artifacts, and increase the resolution of fine details and intricate structures. ${ }^{47}$

Use of model-based or iterative image reconstruction, using a soft reconstruction kernel instead of a sharp kernel, and reconstruction of the images from the acquired raw data with thicker slices reduce the visual conspicuity of metal artifacts. ${ }^{44}$ Iterative reconstruction algorithms apply a larger quantity of acquired data and include photon statistics in the reconstruction, analyzed by intelligent extrapolations. This in theory results in minimized scatter and reduced edge effects by using dedicated correction algorithms.

Visualization of tissues around the metal hardware may be effectively improved by the use of an extended Hounsfield unit scale. $^{12}$

\section{Advanced Techniques of CT Metal Artifact Reduction}

\section{Dual-energy CT with Monochromatic Extrapolation}

Dual-energy computed tomography (DECT) imaging reconstructs images acquired with two photon spectra at different tube voltages, for example, 90 and $140 \mathrm{kV}$. This is achieved simultaneously either by having two separate tubes for each specified $\mathrm{kV}$, fast $\mathrm{kV}$-switching of tube voltage, using beamsplit filters, or operating dual-layer detectors. This is followed by using virtual extrapolated monoenergetic analysis to better evaluate different tissues adjacent to one another, minimize artifacts, and evaluate structures close to implants, in addition to the actual implants at various extrapolated monochromatic energies. ${ }^{48}$

Virtual monochromatic imaging reduces beam hardening artifacts. Optimal monochromatic energies vary for different kinds of metal hardware and tissue type, but most range between 90 and $190 \mathrm{keV}$ (-Figs. 11 and 12). DECT, therefore, enables the reader to evaluate each tissue type at its optimal energy level, without the need for additional imaging. ${ }^{49,50}$

DECT artifact reduction proficiency is inversely proportional to increasing molecular weight of the metal, larger
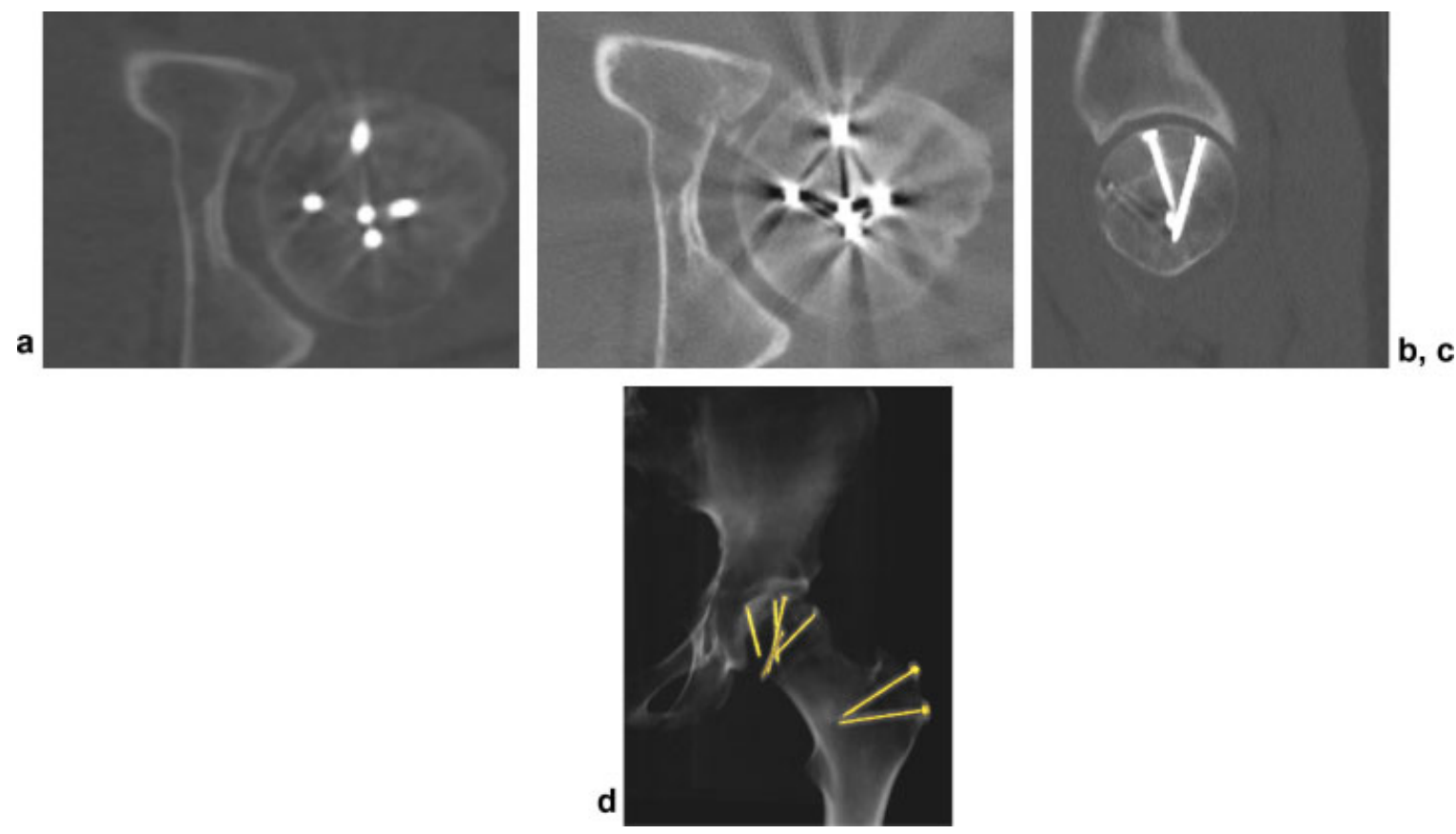

Fig. 11 Computed tomography (CT) examination following greater trochanteric osteotomy and slipped capital femoral epiphysis screw fixation. The visualized effects of metal artifacts are often reduced when evaluating bone window images with (a) smooth kernel image reconstruction rather than (b) sharp kernel image reconstruction. (c) Sagittal virtual monoenergetic reconstruction at 190 keV shows the value of metal artifact reduction using dual-energy CT monoenergetic evaluation for the assessment of screw integrity, location of the screws in relation to the articular surface, extension into the joint space, and contour of the femoral head. (d) Metal artifacts may also be reduced by applying a volume rendering technique to evaluate the integrity of metallic implants. 
a
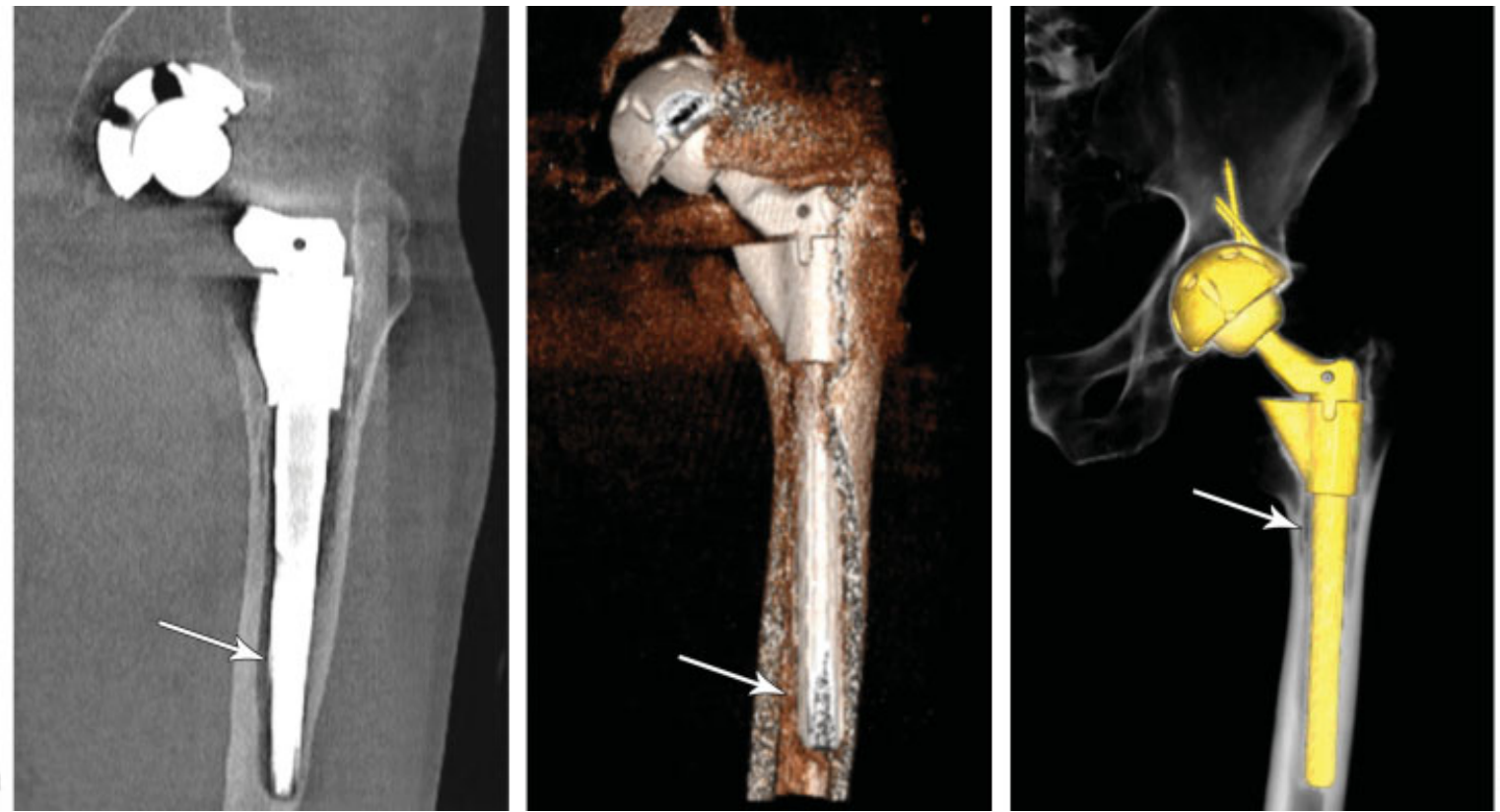

b, c

Fig. 12 Periprosthetic osteolysis of left femoral hip arthroplasty component. (a) Coronal monoenergetic computed tomography (CT) image of the left femur at $180 \mathrm{keV}$, (b) volume rendering CT image of the left femur, and (c) sharp X-ray-type volume rendering CT image demonstrate periprosthetic osteolysis of the femoral component (arrow), varus deformity, and cortical erosion.

volume implants, and metal implants with sharp edges. Switching to higher beam energies might provide some additional artifact reduction; however, in these cases, MAR software is often helpful. ${ }^{51}$

\section{Metal Artifact Reduction Software}

MAR algorithms are based on "projection completion," also known as sinogram inpainting techniques. Simply put, in this class of techniques, the corrupt X-ray projections that traversed the metal hardware are removed and replaced with interpolation from adjacent unaffected projections ( - Fig. 13). Some implementations may also benefit from iterative or model-based reconstructions that additionally incorporate a more realistic model for image acquisition. ${ }^{52}$ Several MAR algorithms were developed in the past. Prototypes include Normalized MAR (NMAR), ${ }^{53}$ Frequency Splitting MAR (FSMAR), ${ }^{54}$ more recently iMAR (iterative metal artifact algorithms that combine the effects of NMAR and FSMAR), adaptive mixing, ${ }^{55,56}$ tissue modeling and adaptive filtering, ${ }^{57}$ and iterative frequency splitting. ${ }^{58}$
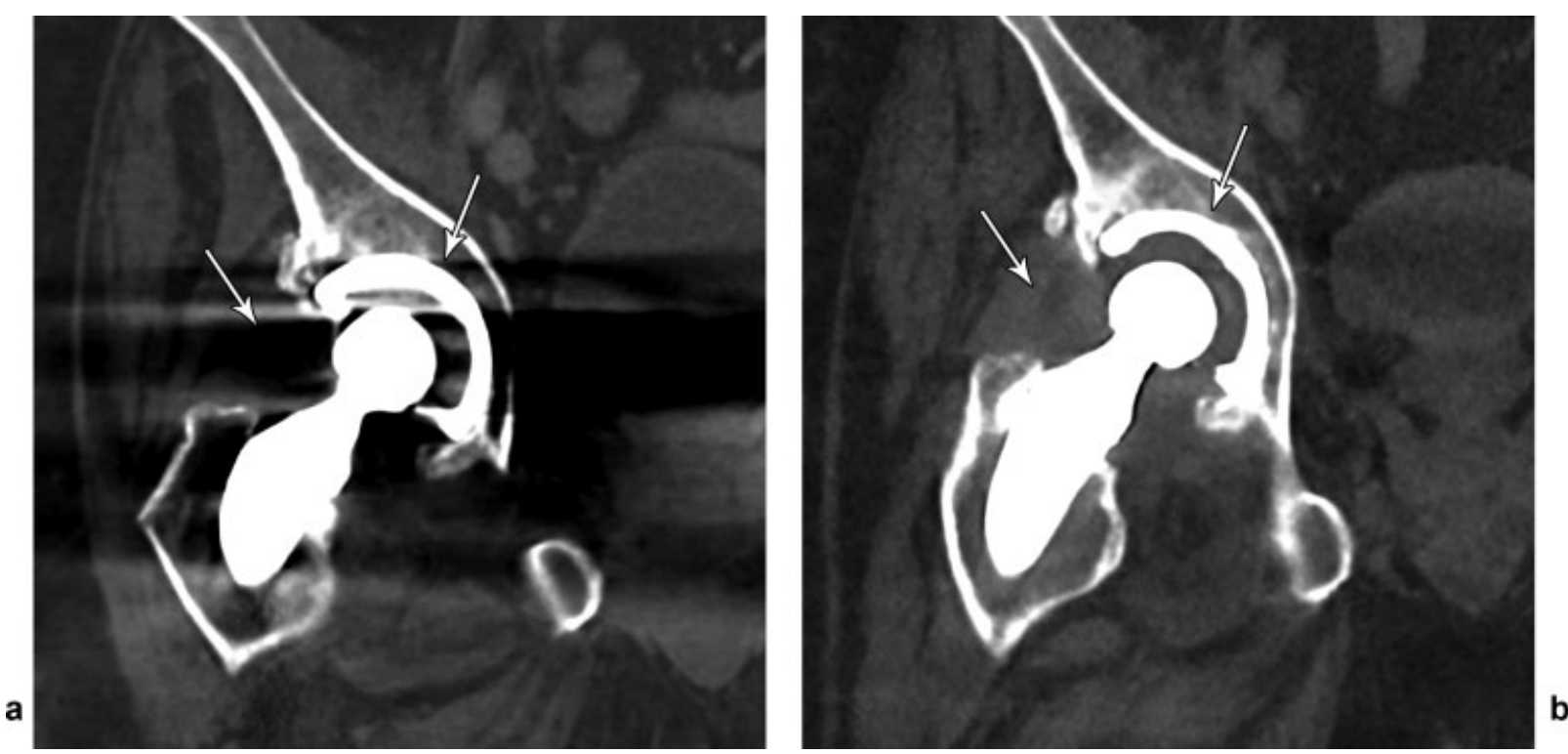

Fig. 13 Total right hip arthroplasty implant in a 71-year-old man. (a) Comparison of conventional filter back-projection computed tomography (CT) image reconstruction and (b) iterative metal artifact reduction CT image reconstruction with inpainting technique demonstrates markedly reduced metal artifacts (arrows) in iterative metal artifact reduction. 
MAR algorithms, despite improving image quality, may obscure parts of the metal hardware, alter the data of areas next to the metal edge, and introduce new artifacts.

Vendors provide MAR software under different commercial names including SEMAR (single-energy MAR, Canon Medical Systems, Otawara, Japan), O-MAR (orthopaedic MAR, Philips Healthcare, Best, Netherlands), SMAR and MARS (Smart MAR and MAR Sequence, respectively, GE Healthcare, Milwaukee, WI), and MARIS and iMAR (MAR in Image Space and iterative MAR, respectively, Siemens Healthineers, Erlangen, Germany). Specific details of these proprietary MAR software are undisclosed. If not already included, the addition of iterative or model-based reconstruction further increases the artifact reduction ability of such software. ${ }^{59}$

\section{Combined MAR Software and Virtual Monochromatic DECT}

The combination of MAR software and virtual monochromatic DECT imaging was advocated in some cases because it may reduce metal-related artifacts more effectively than one technique alone. ${ }^{60-62}$ Limitations to combined use include affecting the appearance of metal implants, over- or underestimating the size of the implant, and maybe even introducing secondary artifacts. ${ }^{61,63,64}$ Depending on the implant composition, size, and shape, no beneficial effects may arise from combining both virtual monochromatic DECT imaging and iterative MAR. ${ }^{65}$

\section{Three-dimensional Postprocessing}

In volume rendering, data integration and averaging from consecutive axial planes into the reformation planes leads to weighing of the true signal over the randomly distributed artifacts that may result in improved image quality and often a visible reduction of metal artifacts ( - Fig. 14) ${ }^{66-68}$

When compared to conventional volume rendering postprocessing techniques, ${ }^{69}$ cinematic rendering is a recently introduced 3D visualization method of volumetric CT and MRI data that applies a highly sophisticated lighting model, enabling the generation of photorealistic images resembling gross anatomical specimens (-Fig. 15). ${ }^{70}$ Sharing a similar concept with volume rendering, cinematic rendering may also reduce tissue obscuration by metal-related artifacts. ${ }^{52}$

\section{Radiation Dose Considerations}

Smart machine designs allow for automated and more accurate estimations of the required X-ray energies and tube currents for optimized CT imaging, based on an individualized patient approach. This helps minimize radiation exposure by ensuring that patients receive the smallest possible dose tolerable for a good quality diagnostic study. ${ }^{71}$

Evolving algorithms allow lower dose CT acquisitions. Utilizing deep learning artificial intelligence methods. CT imaging of THA phantoms using iterative MAR software resulted in the ability to maintain quantitative image quality parameters while reducing CT radiation dose up to $80 \%{ }^{72}$ Furthermore, implementation of an iterative MAR software did not compromise the accuracy of lesion detectability near hardware while reducing CT radiation dose by $50 \% .^{73}$

Carefully constructed DECT protocols ( - Table 3 ) can have comparable effective radiation dose ranges when compared with single-energy CT protocols.
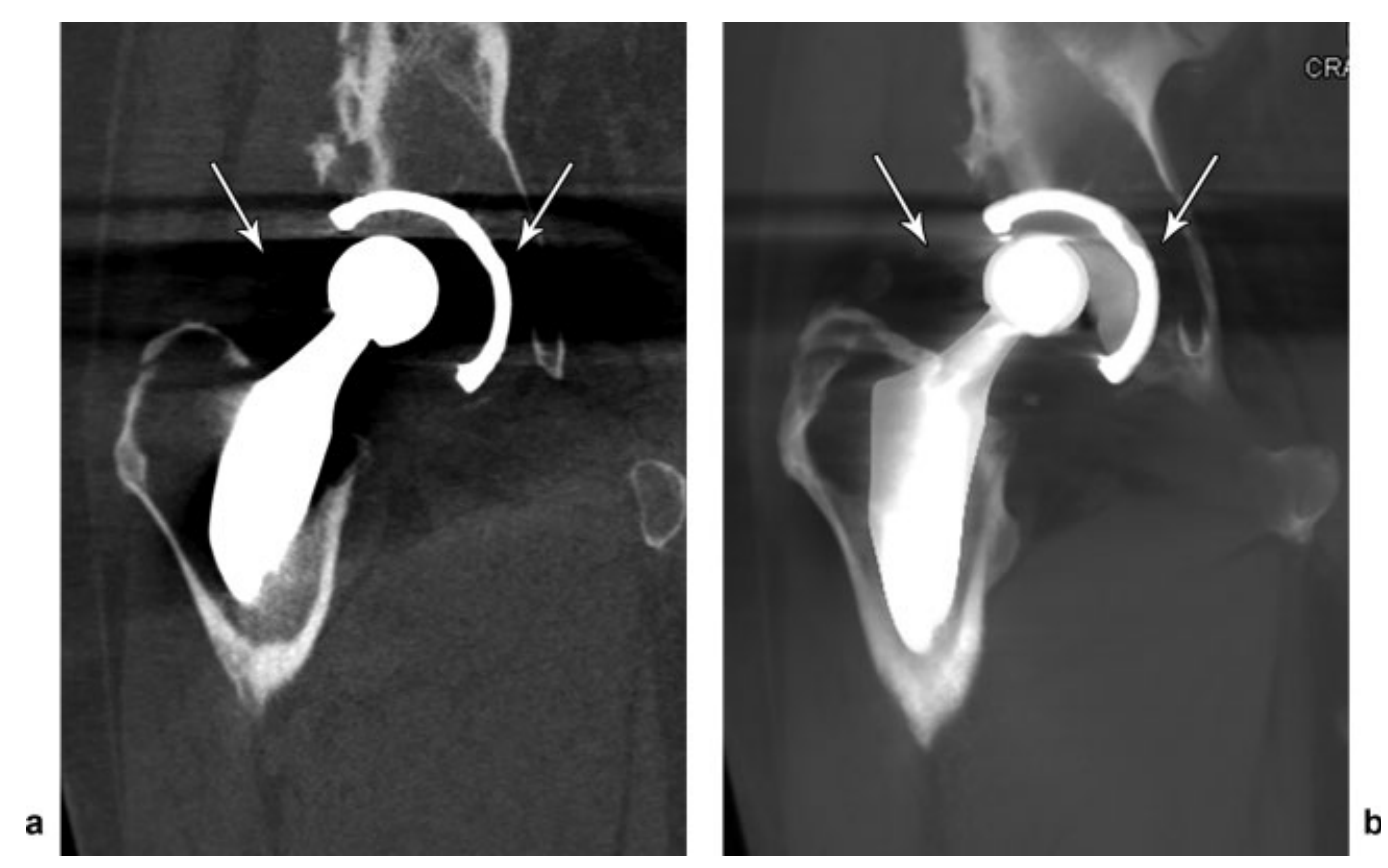

Fig. 14 Total right hip arthroplasty implant in a 78-year-old man with eccentric polyethylene liner wear. Comparison of (a) coronal multiplanar reformation and (b) coronal volume rendering technique computed tomography (CT) images, both created from the same conventional filter back-projection CT data set. Volume rendering technique (b) can result in a visible reduction of low-density metal artifacts (arrows) when compared with conventional multiplanar reformation (a). 

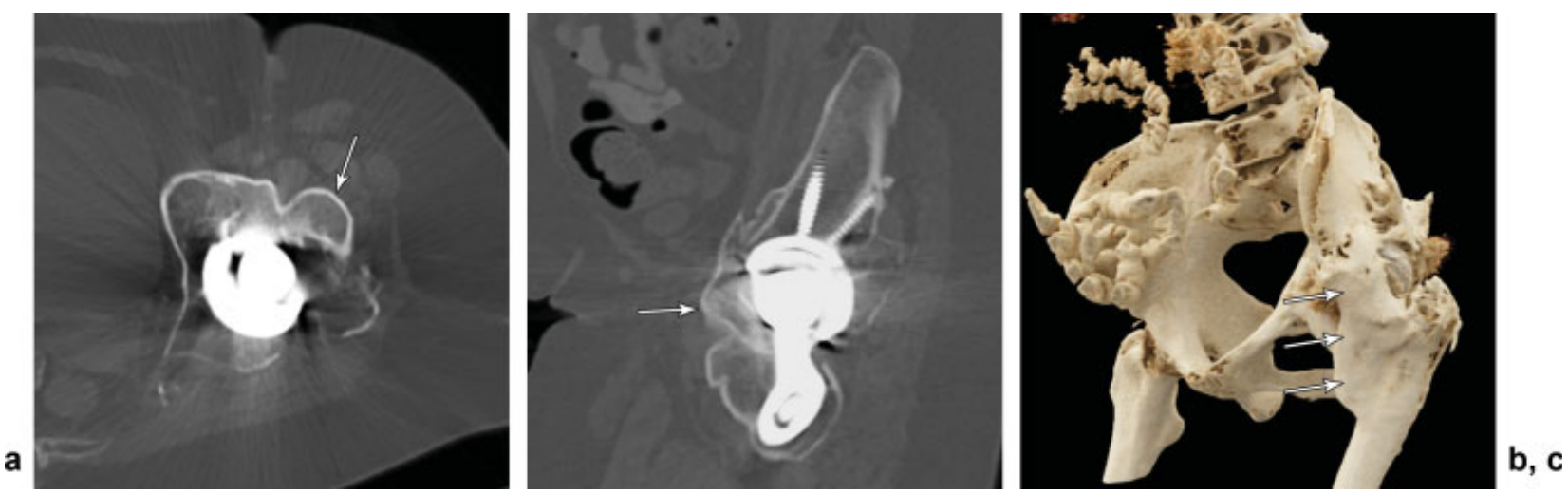

Fig. 15 Total left hip arthroplasty implant in a 59-year-old woman with heterotopic ossification. (a) Axial and (b) sagittal oblique multiplanar reformation computed tomography images demonstrate mature osseous bridging (arrows) between the femur and acetabulum consistent with Brooker class 4 (bone ankylosis), the magnitude of which is visualized to better advantage with cinematic rendering technique (c).

Table 3 Single and dual-energy protocol for CT imaging of hip arthroplasty implants

\begin{tabular}{|l|l|l|}
\hline $\begin{array}{l}\text { Technical } \\
\text { parameter }\end{array}$ & $\begin{array}{l}\text { Value: Single } \\
\text { energy }\end{array}$ & Value: Dual energy \\
\hline Tube energy, kVp & 120 & $\begin{array}{l}\text { 100/Tin (Sn) } \\
\text { filtered 150 }\end{array}$ \\
\hline $\begin{array}{l}\text { Reference tube } \\
\text { current with dose } \\
\text { modulation, mAs }\end{array}$ & 147 & $\begin{array}{l}\text { 300/Adapted to } \\
\text { tube A dose } \\
\text { modulation }\end{array}$ \\
\hline Rotation time, s & 0.5 & 0.5 \\
\hline Collimation & $192 \times 0.6 \mathrm{~mm}$ & $2 \times 192 \times 0.6 \mathrm{~mm}$ \\
\hline Pitch & 0.8 & 0.5 \\
\hline $\begin{array}{l}\text { Average } \\
\text { acquisition time, s }\end{array}$ & 3 & 6 \\
\hline
\end{tabular}

\section{Conclusion}

Rapid advances in metal implant designs and MAR techniques in CT and MRI, as well as evolving patient selection criteria, have significantly improved the visualization and diagnosis of hip arthroplasty implant-related abnormalities that not long ago would have been otherwise undetectable. In clinical practice, an imaging protocol composed of optimized conventional and advanced MAR pulse sequences enables substantial artifact reduction in clinically reasonable acquisition times. DECT with virtual monoenergetic reconstruction, MAR algorithms, or their conjoint implementation can remarkably reduce metal artifacts and improve diagnostic image quality in most cases. Due to the different nature of post-acquisition data analysis and interpolations, CT images reconstructed using MAR algorithms should be interpreted cautiously and in line with the original data sets. New innovations in optimizing imaging aim at refining the algorithms for both CT and MR to further reduce artifacts and adapt to new metal implant designs while aspiring for reductions in acquisition times for MRI and radiation dose for CT.

Conflict of Interest

None declared.

\section{References}

1 Kurtz S, Ong K, Lau E, Mowat F, Halpern M. Projections of primary and revision hip and knee arthroplasty in the United States from 2005 to 2030. J Bone Joint Surg Am 2007;89(04):780-785

2 Patel A, Pavlou G, Mújica-Mota RE, Toms AD. The epidemiology of revision total knee and hip arthroplasty in England and Wales: a comparative analysis with projections for the United States. A study using the National Joint Registry dataset. Bone Joint J 2015; 97-B(08):1076-1081

3 Khodarahmi I, Fritz J. Advanced MR imaging after total hip arthroplasty: The clinical impact. Semin Musculoskelet Radiol 2017;21(05):616-629

4 Hwang SK. Experience of complications of hip arthroplasty. Hip Pelvis 2014;26(04):207-213

5 Graf H, Lauer UA, Berger A, Schick F. RF artifacts caused by metallic implants or instruments which get more prominent at $3 \mathrm{~T}$ : an in vitro study. Magn Reson Imaging 2005;23(03):493-499

6 Graf H, Steidle G, Martirosian P, Lauer UA, Schick F. Metal artifacts caused by gradient switching. Magn Reson Med 2005;54(01): 231-234

7 Graf H, Steidle G, Martirosian P, Lauer UA, Schick F. Effects on MRI due to altered rf polarization near conductive implants or instruments. Med Phys 2006;33(01):124-127

8 Khodarahmi I, Nittka M, Fritz J. Leaps in technology: advanced MR imaging after total hip arthroplasty. Semin Musculoskelet Radiol 2017;21(05):604-615

9 Dillenseger JP, Molière S, Choquet P, Goetz C, Ehlinger M, Bierry G. An illustrative review to understand and manage metal-induced artifacts in musculoskeletal MRI: a primer and updates. Skeletal Radiol 2016;45(05):677-688

10 Hargreaves BA, Worters PW, Pauly KB, Pauly JM, Koch KM, Gold GE. Metal-induced artifacts in MRI. AJR Am J Roentgenol 2011; 197(03):547-555

11 Ahlawat S, Stern SE, Belzberg AJ, Fritz J. High-resolution metal artifact reduction MR imaging of the lumbosacral plexus in patients with metallic implants. Skeletal Radiol 2017;46(07):897-908

12 Lee MJ, Kim S, Lee SA, et al. Overcoming artifacts from metallic orthopedic implants at high-field-strength MR imaging and multi-detector CT. Radiographics 2007;27(03):791-803

13 Kumar NM, de Cesar Netto C, Schon LC, Fritz J. Metal artifact reduction magnetic resonance imaging around arthroplasty implants: the negative effect of long echo trains on the implant-related artifact. Invest Radiol 2017;52(05):310-316

14 Del Grande F, Santini F, Herzka DA, et al. Fat-suppression techniques for 3-T MR imaging of the musculoskeletal system. Radiographics 2014;34(01):217-233

15 Ulbrich EJ, Sutter R, Aguiar RF, Nittka M, Pfirrmann CW. STIR sequence with increased receiver bandwidth of the inversion 
pulse for reduction of metallic artifacts. AJR Am J Roentgenol 2012;199(06):W735-W742

16 Fritz J, Lurie B, Potter HG. MR imaging of knee arthroplasty implants. Radiographics 2015;35(05):1483-1501

17 Molière S, Dillenseger JP, Ehlinger M, Kremer S, Bierry G. Comparative study of fat-suppression techniques for hip arthroplasty MR imaging. Skeletal Radiol 2017;46(09):1209-1217

18 Müller GM, Månsson S, Müller MF, et al. MR imaging with metal artifact-reducing sequences and gadolinium contrast agent in a case-control study of periprosthetic abnormalities in patients with metal-on-metal hip prostheses. Skeletal Radiol 2014;43 (08):1101-1112

19 Cho ZH, Kim DJ, Kim YK. Total inhomogeneity correction including chemical shifts and susceptibility by view angle tilting. Med Phys 1988;15(01):7-11

20 Butts K, Pauly JM, Gold GE. Reduction of blurring in view angle tilting MRI. Magn Reson Med 2005;53(02):418-424

21 Hilgenfeld T, Prager M, Schwindling FS, et al. MSVAT-SPACE-STIR and SEMAC-STIR for reduction of metallic artifacts in 3T head and neck MRI. AJNR Am J Neuroradiol 2018;39(07):1322-1329

22 Ai T, Padua A, Goerner F, et al. SEMAC-VAT and MSVAT-SPACE sequence strategies for metal artifact reduction in 1.5T magnetic resonance imaging. Invest Radiol 2012;47(05):267-276

23 Lu W, Pauly KB, Gold GE, Pauly JM, Hargreaves BA. SEMAC: Slice Encoding for Metal Artifact Correction in MRI. Magn Reson Med 2009;62(01):66-76

24 Chen CA, Chen W, Goodman SB, et al. New MR imaging methods for metallic implants in the knee: artifact correction and clinical impact. J Magn Reson Imaging 2011;33(05):1121-1127

25 Sutter R, Ulbrich EJ, Jellus V, Nittka M, Pfirrmann CW. Reduction of metal artifacts in patients with total hip arthroplasty with sliceencoding metal artifact correction and view-angle tilting MR imaging. Radiology 2012;265(01):204-214

26 Jungmann PM, Bensler S, Zingg P, Fritz B, Pfirrmann CW, Sutter R. Improved visualization of juxtaprosthetic tissue using metal artifact reduction magnetic resonance imaging: experimental and clinical optimization of compressed sensing SEMAC. Invest Radiol 2019;54(01):23-31

27 Fritz J, Lurie B, Miller TT. Imaging of hip arthroplasty. Semin Musculoskelet Radiol 2013;17(03):316-327

28 Fritz J, Lurie B, Miller TT, Potter HG. MR imaging of hip arthroplasty implants. Radiographics 2014;34(04):E106-E132

29 Koch KM, Lorbiecki JE, Hinks RS, King KF. A multispectral threedimensional acquisition technique for imaging near metal implants. Magn Reson Med 2009;61(02):381-390

30 Koch KM, Brau AC, Chen W, et al. Imaging near metal with a MAVRIC-SEMAC hybrid. Magn Reson Med 2011;65(01):71-82

31 Hargreaves BA, Taviani V, Litwiller DV, Yoon D. 2D multi-spectral imaging for fast MRI near metal. Magn Reson Med 2018;79(02): 968-973

32 Hargreaves BA, Chen W, Lu W, et al. Accelerated slice encoding for metal artifact correction. J Magn Reson Imaging 2010;31(04): 987-996

33 Worters PW, Sung K, Stevens KJ, Koch KM, Hargreaves BA. Compressed-sensing multispectral imaging of the postoperative spine. J Magn Reson Imaging 2013;37(01):243-248

34 de Cesar Netto C, Fonseca LF, Fritz B, et al. Metal artifact reduction MRI of total ankle arthroplasty implants. Eur Radiol 2018;28(05): 2216-2227

35 Fritz J, Fritz B, Thawait GK, et al. Advanced metal artifact reduction MRI of metal-on-metal hip resurfacing arthroplasty implants: compressed sensing acceleration enables the time-neutral use of SEMAC. Skeletal Radiol 2016;45(10): 1345-1356

36 Fritz J, Ahlawat S, Demehri S, et al. Compressed Sensing SEMAC: 8fold accelerated high resolution metal artifact reduction MRI of cobalt-chromium knee arthroplasty implants. Invest Radiol 2016; 51(10):666-676
37 Otazo R, Nittka M, Bruno M, et al. Sparse-SEMAC: rapid and improved SEMAC metal implant imaging using SPARSE-SENSE acceleration. Magn Reson Med 2017;78(01):79-87

38 Gotman I. Characteristics of metals used in implants. J Endourol 1997;11(06):383-389

39 Boas F, Fleischmann D. CT artifacts: causes and reduction techniques. Imaging Med 2012;4:229-240

40 Lalonde A, Simard M, Remy C, Bär E, Bouchard H. The impact of dual- and multi-energy $\mathrm{CT}$ on proton pencil beam range uncertainties: a Monte Carlo study. Phys Med Biol 2018;63(19): 195012

41 Park HS, Chung YE, Seo JK. Computed tomographic beam-hardening artefacts: mathematical characterization and analysis. Philos Trans A Math Phys. Eng Sci 2015;373(2043):

42 Joseph PM, Spital RD. The effects of scatter in x-ray computed tomography. Med Phys 1982;9(04):464-472

43 Park HS, Choi JK, Seo JK. Characterization of metal artifacts in Xray computed tomography. Comm Pure Appl Math 2017; 70:2191-2217

44 Wellenberg RHH, Hakvoort ET, Slump CH, Boomsma MF, Maas M, Streekstra GJ. Metal artifact reduction techniques in musculoskeletal CT-imaging. Eur J Radiol 2018;107:60-69

45 Katsura M, Sato J, Akahane M, Kunimatsu A, Abe O. Current and novel techniques for metal artifact reduction at $\mathrm{CT}$ : practical guide for radiologists. Radiographics 2018;38(02): 450-461

46 Mazin SR, Star-Lack J, Bennett NR, Pelc NJ. Inverse-geometry volumetric CT system with multiple detector arrays for wide field-of-view imaging. Med Phys 2007;34(06):2133-2142

47 Barrett JF, Keat N. Artifacts in CT: recognition and avoidance. Radiographics 2004;24(06):1679-1691

48 Gjesteby L, Man BD, Jin Y, Paganetti H, Verburg J, Giantsoudi D, et al. Metal artifact reduction in CT: where are we after four decades? IEEE 2016;4:5826-5849

49 Boudabbous S, Arditi D, Paulin E, Syrogiannopoulou A, Becker C Montet X. Model-Based Iterative Reconstruction (MBIR) for the reduction of metal artifacts on CT. AJR Am J Roentgenol 2015;205 (02):380-385

50 Lewis M, Reid K, Toms AP. Reducing the effects of metal artefact using high keV monoenergetic reconstruction of dual energy CT (DECT) in hip replacements. Skeletal Radiol 2013;42(02): 275-282

51 Higashigaito K, Angst F, Runge VM, Alkadhi H, Donati OF. Metal artifact reduction in pelvic computed tomography with hip prostheses: comparison of virtual monoenergetic extrapolations from dual-energy computed tomography and an iterative metal artifact reduction algorithm in a phantom study. Invest Radiol 2015;50(12):828-834

52 Khodarahmi I, Fishman EK, Fritz J. Dedicated CT and MRI techniques for the evaluation of the postoperative knee. Semin Musculoskelet Radiol 2018;22(04):444-456

53 Meyer E, Raupach R, Lell M, Schmidt B, Kachelriess M. Normalized metal artifact reduction (NMAR) in computed tomography. Med Phys 2010;37(10):5482-5493

54 Meyer E, Raupach R, Lell M, Schmidt B, Kachelrieß M. Frequency split metal artifact reduction (FSMAR) in computed tomography. Med Phys 2012;39(04):1904-1916

55 Mahnken AH, Raupach R, Wildberger JE, et al. A new algorithm for metal artifact reduction in computed tomography: in vitro and in vivo evaluation after total hip replacement. Invest Radiol 2003;38 (12):769-775

56 Liu PT, Pavlicek WP, Peter MB, Spangehl MJ, Roberts CC, Paden RG Metal artifact reduction image reconstruction algorithm for $\mathrm{CT}$ of implanted metal orthopedic devices: a work in progress. Skeletal Radiol 2009;38(08):797-802

57 Bal M, Spies L. Metal artifact reduction in CT using tissue-class modeling and adaptive prefiltering. Med Phys 2006;33(08): 2852-2859 
58 Morsbach F, Bickelhaupt S, Wanner GA, Krauss A, Schmidt B, Alkadhi H. Reduction of metal artifacts from hip prostheses on CT images of the pelvis: value of iterative reconstructions. Radiology 2013;268(01):237-244

59 Wellenberg RH, Boomsma MF, van Osch JA, et al. Computed tomography imaging of a hip prosthesis using iterative model-based reconstruction and orthopaedic metal artefact reduction: a quantitative analysis. J Comput Assist Tomogr 2016;40(06):971-978

60 Bongers MN, Schabel C, Thomas C, et al. Comparison and combination of dual-energy- and iterative-based metal artefact reduction on hip prosthesis and dental implants. PLoS One 2015;10 (11): 0143584

61 Han SC, Chung YE, Lee YH, Park KK, Kim MJ, Kim KW. Metal artifact reduction software used with abdominopelvic dualenergy CT of patients with metal hip prostheses: assessment of image quality and clinical feasibility. AJR Am J Roentgenol 2014; 203(04):788-795

62 Andersson KM, Norrman E, Geijer H, et al. Visual grading evaluation of commercially available metal artefact reduction techniques in hip prosthesis computed tomography. Br J Radiol 2016;89 (1063):20150993

63 Dabirrahmani D, Magnussen J, Appleyard RC. Dual-energy computed tomography-how accurate is gemstone spectrum imaging metal artefact reduction? Its application to orthopedic metal implants. J Comput Assist Tomogr 2015;39(06):925-935

64 Pessis E, Sverzut JM, Campagna R, Guerini H, Feydy A, Drapé JL. Reduction of metal artifact with dual-energy CT: virtual monospectral imaging with fast kilovoltage switching and metal artifact reduction software. Semin Musculoskelet Radiol 2015;19 (05):446-455
65 Khodarahmi I, Haroun RR, Lee M, et al. Metal artifact reduction computed tomography of arthroplasty implants: effects of combined modeled iterative reconstruction and dual-energy virtual monoenergetic extrapolation at higher photon energies. Invest Radiol 2018;53(12):728-735

66 Zhang X, Wang J, Xing L. Metal artifact reduction in X-ray computed tomography (CT) by constrained optimization. Med Phys 2011;38(02):701-711

67 Zhang X, Xing L. Sequentially reweighted TV minimization for CT metal artifact reduction. Med Phys 2013;40(07):071907

68 Zhang Y, Yan H, Jia X, Yang J, Jiang SB, Mou X. A hybrid metal artifact reduction algorithm for X-ray CT. Med Phys 2013;40(04): 041910

69 Fritz J, Fishman EK, Corl F, Carrino JA, Weber KL, Fayad LM. Imaging of limb salvage surgery. AJR Am J Roentgenol 2012;198 (03):647-660

70 Rowe SP, Fritz J, Fishman EK. CT evaluation of musculoskeletal trauma: initial experience with cinematic rendering. Emerg Radiol 2018;25(01):93-101

71 Seo N, Chung YE, An C, Choi JY, Park MS, Kim MJ. Feasibility of radiation dose reduction with iterative reconstruction in abdominopelvic CT for patients with inappropriate arm positioning. PLoS One 2018;13(12):e0209754

72 Wellenberg RH, Boomsma MF, van Osch JA, et al. Low-dose CT imaging of a total hip arthroplasty phantom using model-based iterative reconstruction and orthopedic metal artifact reduction. Skeletal Radiol 2017;46(05):623-632

73 Subhas N, Pursyko CP, Polster JM, et al. Dose reduction with dedicated CT metal artifact reduction algorithm: CT phantom study. AJR Am J Roentgenol 2018;210(03):593-600 